Special issue of the International Conference on Computational and Experimental Science and Engineering (ICCESEN 2014)

\title{
Orthogonalizing Boolean Subtraction of Minterms or Ternary-Vectors
}

\begin{abstract}
Y. CAN* AND G. FisCHER
Friedrich-Alexander University Erlangen-Nuremberg, Institute for Technical Electronics, Erlangen, Germany

In this paper, a method called "Boolean subtraction" which enables the subtraction of two minterms and of two functions of the disjunctive normal form respectively of two ternary-vectors and of two ternary-vector-lists is presented. The advantage of "Boolean subtraction" is that the calculated results are already presented in an orthogonal form, which has a significant advantage for further calculations. It replaces two procedures, once building the difference and then the subsequent orthogonalizing and has faster computing time in comparison. Furthermore, another equation is developed from the property of this new method providing orthogonal results in order to orthogonalize functions of the disjunctive normal form.
\end{abstract}

DOI: 10.12693/APhysPolA.128.B-388

PACS: $02.10 . \mathrm{Ab}$

\section{Introduction}

In contrast to the Boolean operators AND ' $\wedge$ ', OR ' $\vee$ ', NOT ' $\neg$ ', XOR ' $\oplus$ ' and XNOR ' $\odot$ ' [1], subtraction and division are not defined in the Boolean algebra. This work shows a method of subtracting two minterms, two functions respectively two ternary-vectors (TV), two ternary-vector-lists (TVL) of the disjunctive normal form $D(f)$. A special feature of this method is the result of the difference, which has the property of being orthogonalized. The "Boolean subtraction" shown here does not correspond to the subtraction in algebra, in which a number is subtracted from another number. It is more like the set difference in the set theory, whereas the result of "Boolean subtraction" is already orthogonalized. By the "Boolean subtraction $\ominus$ " not a new operator is introduced, but rather a new function which orthogonalizes. This subtraction is illustrated by using the Karnaugh map. Based on this method, a new mathematical equation to orthogonalize functions of the disjunctive normal form easily is going to be formed.

\section{Theoretical foundations}

\subsection{Isomorphism: from the set theory to boolean algebra}

In Fig. 1 the set difference from the set theory [2] is shown and defined by:

$$
C=A \backslash B=\{x \mid(x \in A) \wedge(x \notin B)\} .
$$

Due to the isomorphism the set difference is expressed by Eq. (2). Thus, the difference of two minterms $m_{i, j}$, respectively of two ternary-vectors $T V_{i, j}$ can be calculated:

$$
C=A \backslash B=A \cap \bar{B} \rightarrow A \wedge \bar{B} .
$$

\footnotetext{
*corresponding author; e-mail: yavuz.can@fau.de
}

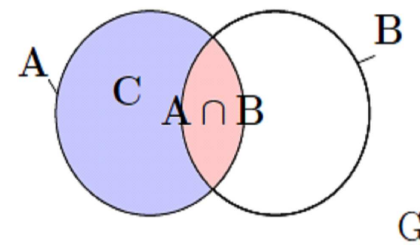

Fig. 1. $\mathrm{A} \backslash \mathrm{B}$ in Venn diagram.

\subsection{Ternary-vector-list}

Ternary-vector-lists (TVLs) are representations of Boolean functions which may assume one of three possible values for each point. A non-negated variable is characterized by ' 1 ', a negated by ' 0 ' and a variable not included by '-'. TVLs can be treated computationally easier and are more advantageous in terms of memory requirement and computing time. A TVL consists of $m$-rows (number of conjunctions or disjunctions contained in the function), called ternary-vector (TV), and $n$-columns (number of independent variables) and with $t \in\{0,1,-\}$ and $n, m \in \mathbb{N}$ applies (Eq. (3)) [3-5]:

$$
\begin{gathered}
T V L=\left[\begin{array}{c}
T V_{1} \\
T V_{2} \\
\vdots \\
T V_{m}
\end{array}\right]=\left[\begin{array}{cccc}
x_{n-1} & \ldots & x_{1} & x_{0} \\
t_{1(n-1)} & \ldots & t_{11} & t_{10} \\
t_{2(n-1)} & \ldots & t_{21} & t_{20} \\
\vdots & \ldots & \vdots & \vdots \\
t_{m(n-1)} & \ldots & t_{m 1} & t_{m 0}
\end{array}\right]= \\
{\left[t_{m(n-1)}\right] .}
\end{gathered}
$$

The rule for the AND-operation $(\wedge)$ of two ternaryvectors $T V_{i, j}$, which represents an average, is defined by the following way:

\begin{tabular}{|c|c|c|c|}
\hline$\wedge$ & 0 & 1 & - \\
\hline 0 & 0 & $\times$ & 0 \\
\hline 1 & $\times$ & 1 & 1 \\
\hline- & 0 & 1 & - \\
\hline
\end{tabular}


Example 1: The block of $2([-111])$ is subtracted from a block of $8([1---])$, see Fig. 2 .

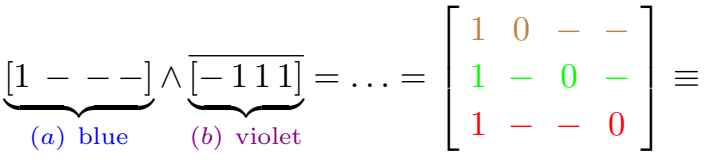

$$
\begin{aligned}
& \underbrace{x_{3} \bar{x}_{2}}_{\text {brown }} \wedge \underbrace{x_{3} \bar{x}_{1}}_{\text {green }} \wedge \underbrace{x_{3} \bar{x}_{0}}_{\text {red }} .
\end{aligned}
$$

The result is a TVL of three TVs, blocks of 4 (red, brown, green), which cover the rest of the $1 \mathrm{~s}$ with the largest possible covering and overlap themselves (Fig. 2, $(a \backslash b)$ ).

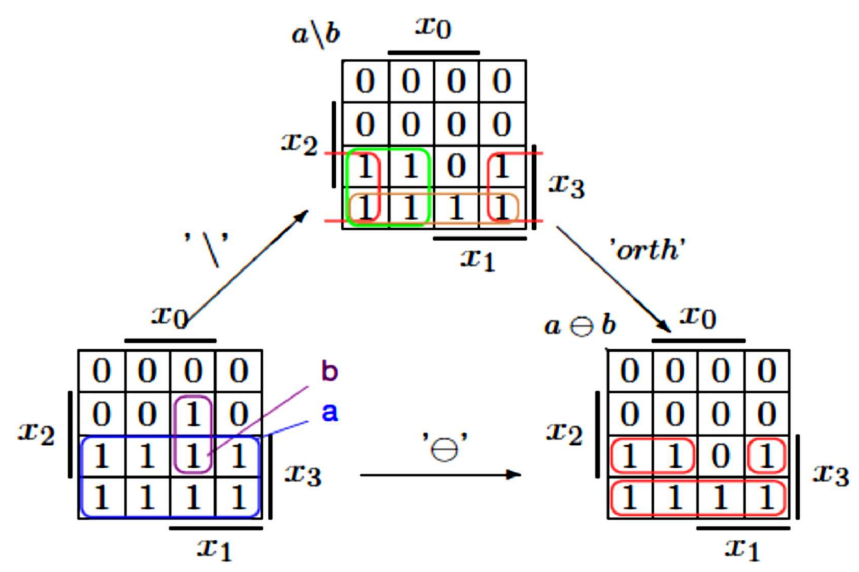

Fig. 2. ' $\ominus$ ': composition of ' $\backslash$ ' and 'orth'.

Orthogonality: A TVL is orthogonal if the TVs have the characteristic to be disjoint to each other in pairs at least in one column. Thus $T V_{i} \wedge T V_{j}=\times$ can be formulated for the orthogonality of a TVL [4]. The orthogonality of a TVL respectively function of the disjunctive normal form is characterized in a $K$-map by non-overlapping blocks. Orthogonal TVLs can be used as internal computer representations for binary functions and are more advantageous for further calculations.

\section{The Boolean subtraction}

Subtraction is one of the four basic operations in arithmetics in which a number (subtrahend) is subtracted from another number (minuend), and is defined as: Minuend - Subtrahend = Difference. Although does not work in the same way the method of "Boolean subtraction" to determine the difference as in arithmetics, the same terminology is used to explain this new function.

The "Boolean subtraction" is illustrated by the same two blocks of Example 1 in a $K$-map with 4 variables (Fig. 3). It is the removal of the intersection set from the minuend that arises between the minuend and the subtrahend. After the removal of a subtrahend from a minuend a difference which includes several blocks, which are pairwise orthogonal to each other and cover the remaining 1 s of the minuend arises:

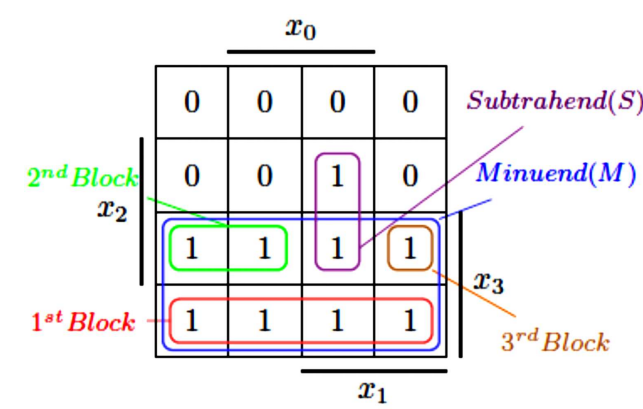

Fig. 3. Illustration of Boolean subtraction in $K$-map.

$$
x_{3} \ominus x_{2} x_{1} x_{0}=\underbrace{x_{3} \bar{x}_{2} \vee x_{3} x_{2} \bar{x}_{1} \vee x_{3} x_{2} x_{1} \bar{x}_{0}}_{\text {blocks of difference }}
$$

The corresponding TVL representation:

$$
[1---] \ominus[-111]=\left[\begin{array}{cccc}
1 & 0 & - & - \\
1 & 1 & 0 & - \\
1 & 1 & 1 & 0
\end{array}\right] \text {. }
$$

The number of the variables abounded in the subtrahend and are not presented in the minuend at the same time corresponds to the number of blocks in the result (called $n$ ). The result is dependent on the starting variable. There are several options, but all of them are homogeneous because the same set of $1 \mathrm{~s}$ is covered. They differ only in the form of coverage. The set of $1 \mathrm{~s}$ is be covered by the largest possible blocks which are orthogonal. The number of possible results can be determined by: $n$ ! for $n>0$. Equation (4) is applied to calculate the "Boolean subtraction" of two minterms or two ternaryvectors. If the subtrahend and the minuend are orthogonal to each other, the result corresponds to the minuend itself.

$$
\begin{aligned}
& m_{m} \ominus m_{S}=\left(x_{n-1} \wedge \ldots \wedge x_{1} \wedge x_{0}\right)_{m} \\
& \ominus\left(x_{n-1} \wedge \ldots \wedge x_{1} \wedge x_{0}\right)_{S}:= \\
& {\left[\left(x_{n-1} \wedge \ldots \wedge x_{1} \wedge x_{0}\right)_{m} \wedge \overline{x_{n-1}}\right] \vee \ldots \vee} \\
& {\left[\left(x_{n-1} \wedge \ldots \wedge x_{1} \wedge x_{0}\right)_{m} \wedge \overline{x_{n-2}} \wedge x_{n-1}\right] \vee \ldots \vee} \\
& {\left[\left(x_{n-1} \wedge \ldots \wedge x_{1} \wedge x_{0}\right)_{m}\right.} \\
& \left.\wedge \overline{x_{1}} \wedge \ldots \wedge x_{n-2} \wedge x_{n-1}\right] \vee \\
& {\left[\left(x_{n-1} \wedge \ldots \wedge x_{1} \wedge x_{0}\right)_{m}\right.} \\
& \left.\wedge \overline{x_{0}} \wedge x_{1} \wedge \ldots \wedge x_{n-2} \wedge x_{n-1}\right] .
\end{aligned}
$$

By the new method the two calculation procedures, the difference building 'Y' and the subsequent orthogonalization 'orth' [6] are performed in one step (Fig. 2), which means the "Boolean subtraction" is the composition of both. The orthogonalization of a TVL is reached by filling the [-]-column with the complement of the variable in 


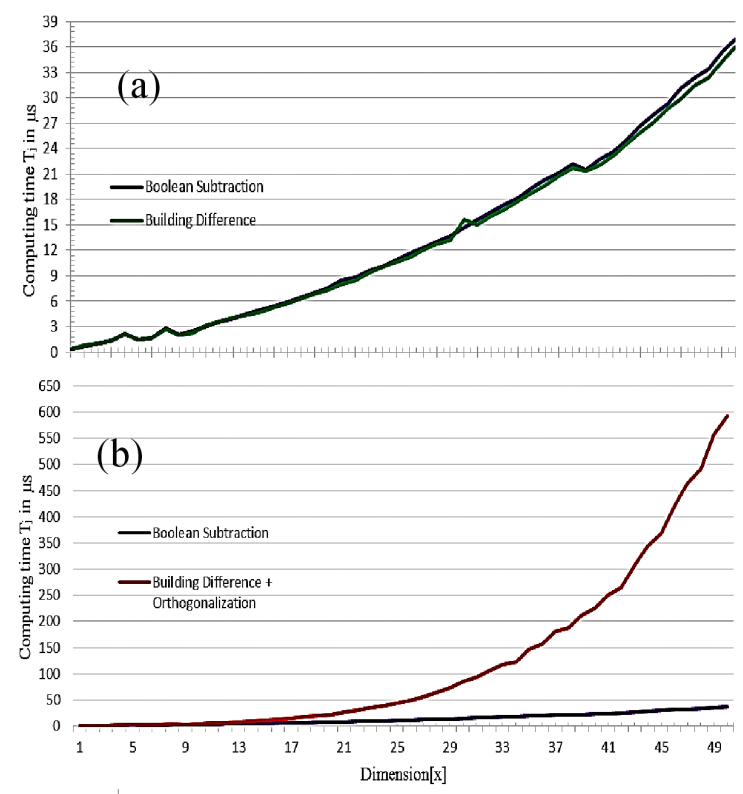

Fig. 4. (a) comparison ' $\ominus$ ' vs. ' $\backslash$ ', (b) comparison of ' $\ominus$ ' vs. ' ' and 'orth' with respect to $T_{j}$.

the same column of another TV. In TVL with multiple TVs the calculation of orthogonal solution by the corresponding implementation is much more difficult, whereas the new function constitutes the solution in already orthogonal form.

Both TVL-results, out of building difference and "Boolean subtraction", are different in their TVL representation but equal in the covering the $1 \mathrm{~s}$. The result by is the orthogonal form of the result by building the difference out of the set theory (Fig. 3).
The comparison between "Boolean subtraction" and building the difference is shown in diagram (Fig. 4a), whereas the comparison of "Boolean subtraction" and the composition with respect to the computing time depending on the dimension (Dimension $[x]$ ) is illustrated in diagram (Fig. 4b). For each single dimension, which is the length of the ternary-vector of the minuend and the subtrahend, 1000 different tasks are performed. In addition, for each task is determined an average computing time $T_{i}$ is determined out of 1000 measurements. Consequently, an average computing time $T_{j}\left(T_{j}^{[x]}=\frac{\sum_{i=1}^{1000} \sum_{k=1}^{1000} T_{i k}}{1000 \cdot 1000}\right)$ for each dimension is determined out of the average computing time values $T_{i}$, in order to compensate abnormalities. The curves in the first diagram (Fig. 4a) run almost identically but the second diagram (Fig. 4b) clearly shows that the new method has faster computing time with increasing dimension than it is the case with the composition of building the difference with the subsequent orthogonalization [7].

\subsection{Boolean subtraction of two functions or two TVLs}

The orthogonalized difference of two functions of disjunctive normal form, respectively of two TVLs $D(f)$, can be calculated by Eq. (5) based on "Boolean subtraction". One of them is the orthogonal minuend-function $f_{m}^{o r t h}$ and the other one is the subtrahend-function $f_{S}$. The subtrahend is subducted from the set of the 1s of the orthogonal minuend and the result is represented in an orthogonal form. Eq. (5) can be used for calculating the orthogonal complement of a function by $D(1) \ominus D(f)=D\left(\bar{f}^{\text {orth }}\right)$. That means that the set of 0 s with $D\left(\bar{f}^{\text {orth }}\right)$ corresponds to the total set $D(1)$ without the set of 1 s of $D(f)$

$$
f_{m}^{\text {orth }} \ominus f_{S}=\left[\begin{array}{c}
m_{1 M} \\
m_{2 M} \\
\vdots \\
m_{n M}
\end{array}\right] \ominus\left[\begin{array}{c}
m_{1 S} \\
m_{2 S} \\
\vdots \\
m_{n S}
\end{array}\right]=\left[\begin{array}{c}
\left(m_{1 M} \ominus m_{1 S}\right) \wedge\left(m_{1 M} \ominus m_{2 S}\right) \wedge \ldots \wedge\left(m_{1 M} \ominus m_{n S}\right) \\
\left(m_{2 M} \ominus m_{1 S}\right) \wedge\left(m_{2 M} \ominus m_{2 S}\right) \wedge \ldots \wedge\left(m_{2 M} \ominus m_{n S}\right) \\
\vdots \\
\left(m_{n M} \ominus m_{1 S}\right) \wedge\left(m_{n M} \ominus m_{2 S}\right) \wedge \ldots \wedge\left(m_{n M} \ominus m_{n S}\right)
\end{array}\right]
$$

\section{Orthogonalization using Boolean subtraction}

Functions of disjunctive normal form, $f_{D N F}=m_{1} \vee$ $m_{2} \vee \ldots \vee m_{i}$ and $m_{i}=x_{(n-1)} \cdot \ldots \cdot x_{1} \cdot x_{0}$ with $i \in$ $\mathrm{N}$, can be orthogonalized by Eq. (6) based on "Boolean subtraction". Certainly the orthogonalized solution can diversify depending on the starting minterm; the order of the minterms can be changed because of the commutative characteristic of a disjunctive normal form. The order of the calculation is important. That means, the first two minterms must be subtracted and then the third minterm must be subtracted from the result of them, and so on. If the function is composed of three minterms $(i=3)$, the form $f_{D N F}^{o r t h}=\left[m_{1} \ominus m_{2} \ominus m_{3}\right] \vee\left[m_{2} m_{3}\right] \vee m_{3}$ is derived from Eq. (6)

$$
f_{D N F}^{\text {orth }}=\bigvee_{k=0}^{i-1}\left[\ominus_{n=k+1}^{i} m_{n}\right] .
$$

Example 2: The function

$$
f_{2}=\overline{x_{1}} \vee x_{0} \vee x_{2} \equiv\left[\begin{array}{ccc}
- & 0 & - \\
- & - & 1 \\
1 & - & -
\end{array}\right]
$$

is orthogonalized as follows: 


$$
\begin{gathered}
f_{2}^{\text {orth }}=\left[\begin{array}{c}
\bar{x}_{1} \ominus x_{0} \ominus x_{2} \\
x_{0} \ominus x_{2} \\
x_{2}
\end{array}\right]=\left[\begin{array}{c}
\bar{x}_{1} \bar{x}_{0} \ominus x_{2} \\
\bar{x}_{2} x_{0} \\
x_{2}
\end{array}\right]= \\
{\left[\begin{array}{c}
\bar{x}_{2} \bar{x}_{1} \bar{x}_{0} \\
\bar{x}_{2} x_{0} \\
x_{2}
\end{array}\right] \equiv\left[\begin{array}{lll}
0 & 0 & 0 \\
0 & - & 1 \\
1 & - & -
\end{array}\right]}
\end{gathered}
$$

$f_{2}^{\text {orth }}$ is the orthogonalized form of $f_{2}$. They are different in their form, but homogeneous because they cover the same 1s, which means, they are equivalent in their value. An orthogonal disjunctive normal form is equivalent to an orthogonal antivalence normal form, $D\left(f^{\text {orth }}\right)=A\left(f^{\text {orth }}\right)$. So it can immensely facilitate further calculations such as the "Boolean differential calculus" [6].

\section{Conclusion}

This work shows the new method "Boolean subtraction". The result of this variety of subtraction is a set, which elements are also sets itselves, which are disjoint to each other in pairs. This method can be clearly visualized in a $K$-map up to 4 variables. The "Boolean subtraction" is applied to the subtraction of two minterms respectively of two ternary-vectors, two functions of disjunctive normal form respectively of two ternary-vector-lists $D(f)$. This new function " saves a further step of orthogonalization. TVL of the form $D(f)$ respectively functions of disjunctive normal form can easily be orthogonalized mathematically by the new Eq. (6) based on the new method for orthogonalization. The application of this equation is also enabled in the TVL arithmetic. Due to the orthogonalization further processing steps in the TVL arithmetic are simplified to a large extent because the orthogonal form of the disjunctive normal form has the advantage to assume them to be the antivalence normal form. Thus, it can significantly facilitate further calculations, such as the "Boolean differential calculus".

\section{References}

[1] H.J. Zander, Logischer Entwurf binärer Systeme. 3. bearb. Auflage, Verl. Technik, Berlin 1989.

[2] L. Popula, Mathematik für Ingenieure und Naturwissenschaften, Band 1., 13. durchgelesene Auflage, Viewer und Teubner Verlag - Springer Fachmedien, Wiesbaden 2011.

[3] M. Kühnrich, Dissertation. Technische Hochschule Karl-Marx-Stadt, Chemnitz 1979.

[4] D. Bochmann, Binäre Systeme. Ein BOOLEAN Buch. LiLoLe-Verlag GmbH, Hagen 2006.

[5] G. Kempe, Ph.D. Thesis, Technische Universität Bergakademie, Freiberg 2003.

[6] Ch. Posthoff, Dissertation, Technische Hochschule Karl-Marx-Stadt, Chemnitz 1979.

[7] H. Kassim, M.Sc. Thesis, Institute for Reliable Circuits and Systems, Friedrich-Alexander-University Erlangen-Nürnberg, Germany. 